University of Massachusetts Amherst

ScholarWorks@UMass Amherst

Chemistry Department Faculty Publication Series

Chemistry

1983

\title{
Flow Injection Calibration Methods for Atomic Absorption Spectrometry
}

Julian Tyson

University of Massachusetts Amherst

Follow this and additional works at: https://scholarworks.umass.edu/chem_faculty_pubs

Part of the Chemistry Commons

\section{Recommended Citation}

Tyson, Julian, "Flow Injection Calibration Methods for Atomic Absorption Spectrometry" (1983). Analytica Chimica Acta. 1281.

Retrieved from https://scholarworks.umass.edu/chem_faculty_pubs/1281

This Article is brought to you for free and open access by the Chemistry at ScholarWorks@UMass Amherst. It has been accepted for inclusion in Chemistry Department Faculty Publication Series by an authorized administrator of ScholarWorks@UMass Amherst. For more information, please contact scholarworks@library.umass.edu. 


\title{
FLOW INJECTION CALIBRATION METHODS FOR ATOMIC ABSORP- TION SPECTROMETRY
}

\author{
J. F. TYSON*, J. M. H. APPLETON and A. B. IDRIS \\ Department of Chemistry, Loughborough University of Technology, Loughborough, \\ Leicestershire LE11 3 TU (Gt. Britain)
}

(Received 8th June 1982)

\section{SUMMARY}

The use of an atomic absorption spectrometer as a detector in flow injection analysis is briefly reviewed. A new simplified model is described for the dispersion effects observed with such systems; the model is based on considering the dispersion to be due to a single hypothetical mixing chamber located immediately prior to the measurement stage. The utility of this approach is demonstrated for two methods of calibration commonly used in atomic absorption spectrometry, and it is shown that flow injection sample and standard handling techniques are comparable to manipulation with volumetric apparatus. The flow injection method has a number of advantages for the analogue of the standard addition method. The use of an exponential concentration gradient is proposed as a novel method of calibration using a single concentrated standard. Results are presented for the determination of chromium in standard steels.

Since 1975, when flow injection analysis (f.i.a.), as the term is generally understood, was described by Rủžčka and Hansen [1], there has been considerable interest in applying the elegant simplicity of the basic idea to a wide range of analytical methods. Most of these applications have utilized the chemistries of existing analytical techniques and considerable ingenuity has been used in adapting some of these techniques to continuously flowing systems. The essence of f.i.a. is to allow reproducible dispersion between a plug of sample solution injected into a carrier stream and a reagent in, or added to, the carrier stream so that the extent of the reaction may be monitored by a downstream detector. Thus most applications of f.i.a. have used a system comprising a detector appropriate to monitoring the chemical reaction and spectroscopic (mainly molecular absorption spectrophotometry) and electrochemical (mainly potentiometric) techniques have been prominent. In addition to the great varieties of chemistries adapted for f.i.a., the high precision of which enables some of the stringent kinetic requirements of reactions of analytical utility to be relaxed, it has not been overlooked that the rapid through-put of samples possible with f.i.a. (one of its attractive features as a fully automated system) means that f.i.a. can be used for analytical measurements where no chemistry is required, merely rapid, reproducible transport of sample solution to detector. For such analyses, a low 
dispersion system is required so that unnecessary dilution does not occur, or the dispersion can be designed so that solutions which are "off-range" can be suitably diluted.

There is a tendency for the through-put of a flow injection system to be described in terms of so many samples per hour, although this figure is not synonymous with so many determinations per hour. As current practice favours injection of samples in triplicate, and as allowance must be made for similar injection of the range of calibration standards, a more realistic figure might be obtained by dividing the samples-per-hour figure by three (or more) and calling it determinations per hour. Such a calculation still does not allow for the time spent in obtaining the sample or for any pretreatment it may need to get it into a form suitable for injection.

The use of atomic absorption spectrometry (a.a.s.) as the detection method in f.i.a. has been reported on only about ten occasions. Thus a.a.s. has not excited much interest as a detector system for f.i.a.; indeed, most of the applications would be better described as using flow injection simply as a sample introduction system. Nearly all the applications described use the limited dispersion flow injection mode described above, so that the sample handling system could be considered as an automated "discrete nebulization" [2] or "micro-sampling nebulization" [3] accessory. The advantages of flow injection for sample introduction are exemplified by Wolf and Stewart who report [4] on the excellent precision (hence low detection limits) obtainable for zinc and copper and the improvement in nebulizer performance achieved when the flow rate of carrier is controlled by a suitable pump rather than by the oxidant flow rate.

Nebulizer performance was also considered by Yoza et al. [5] who, in determining magnesium, used a compensating flow of either air or solution to match the manifold flow rate to the optimum flow rate of the nebulizer. The enhancing effects of organic solvents were utilized by Fukamachi and Ishibashi [6] who injected aqueous solutions of a number of metals into a carrier stream of an immiscible solvent (either n-butyl acetate or methyl isobutyl ketone) propelled solely by the "suction" of the nebulizer. Bergamin and co-workers [7-9] have demonstrated the feasibility of their zonesampling injection technique as well as the use of the merging-zone technique for the addition of lanthanum to a calcium solution. The simultaneous use of both dual-channel atomic absorption and dual-channel atomic emission spectrometers enabled Basson and van Staden [10] to analyse water samples for four elements simultaneously by splitting the stream between the two instruments. Mindel and Karlberg [11] have outlined some of the advantageous features of using flow injection as a sample introduction system and suggested that solvent extraction could be carried out in the flowing stream. Tyson and Idris $[12,13]$ have discussed the characteristics of the instrument response curve and demonstrated the feasibility of the flow injection analogue of the standard addition method; they proposed the use of a concentration gradient generated by flow injection as the basis of a single-standard calibra- 
tion method. Greenfield [14] has demonstrated that these ideas are also workable with an inductively-coupled plasma instrument whose use has also been described by Jacintho et al. [15]. Recently, the hydride generation reaction has been adapted to a flow injection manifold by Aström [16], for the determination of bismuth.

\section{Model for dispersion behaviour}

The extent to which a sample plug disperses during its passage through a narrow tube under conditions of laminar flow has been the subject of numerous studies. Exact solution of the appropriate equations appears difficult and various numerical methods have been based on introduction of reduced units to make the system of equations dimensionless. The relevance of these to the conditions normally encountered in flow injection manifolds has recently been critically evaluated [17] and it has been suggested that these numerical solutions are more useful than the solutions obtainable when either convection or diffusion is regarded as the predominant mechanism. The latter solutions have been used as a basis for explaining the dispersion phenomena encountered in f.i.a. and for providing a number of guidelines for the design of manifolds [18].

In an atomic absorption spectrometer, several physical and chemical processes occur in converting the solution flowing into the nebulizer to a population of free atoms. The resultant absorbance-time relationship is, to a good approximation, exponential and thus the atomic absorption instrument behaves as though it contained a single well-stirred mixing chamber. The concentration $(C)$-time $(t)$ relationship when a step change in concentration from 0 to $C_{m}$ occurs in a stream flowing with volume flow rate $u$, just prior to a mixing chamber of volume $V$ is given by

$C=C_{\mathrm{m}}[1-\exp (-u t / V)]$

If it is assumed that absorbance $(A)$ is directly proportional to concentration, then the shape of the corresponding $A-t$ curve is given by

$A=A_{\mathrm{m}}[1-\exp (-u t / V)]$

(All symbols used are explained in Table 1.) The curve shape can be analyzed to obtain a value of $V$ if $A_{\mathrm{m}}$ and $u$ are known. A plot of $\ln \left[A_{\mathrm{m}} /\left(A_{\mathrm{m}}-A\right)\right]$ vs. $t$ has a slope of $u / V$. For typical flow rates, compatible with optimum nebulizer performance, $V$ ranges between 60 and $100 \mu$ l. Thus the flow injection equivalent of the basic a.a.s. manifold is as shown in Fig. 1. It was observed that increasing the length of the connecting tubing, $L$, up to $200 \mathrm{~cm}$ still produced exponentially-shaped peak profiles. Thus it is proposed that a convenient way of describing the total dispersion effects (caused by injection, flow system and detector) is to consider them as due to a hypothetical single mixing chamber located immediately prior to a detector with an instantaneous response. In this model, pure plug flow is considered to occur between the point of injection and the mixing chamber. 
List of symbols

\begin{tabular}{|c|c|}
\hline$A$ & Absorbance \\
\hline$A_{\mathrm{m}}$ & Steady-state absorbance (or infinite volume absorbance) \\
\hline$A_{\mathrm{P}}$ & Peak absorbance \\
\hline$C^{2}$ & Concentration \\
\hline$C_{\mathrm{m}}$ & Steady-state concentration \\
\hline$C_{\mathrm{P}}$ & Peak concentration \\
\hline$C^{\mathbf{R}}$ & Concentration of reagent in carrier stream \\
\hline$C^{\mathrm{S}}$ & Concentration of standard injected $\quad{ }^{\prime} i$ \\
\hline$C_{t}^{S}$ & Concentration of top standard in calibration sequence \\
\hline$C_{x}^{t}$ & Concentration of sample \\
\hline$D$ & Dispersion based on concentration of injected solution, $D=C_{\mathrm{m}} / C_{\mathrm{p}}$ \\
\hline$D^{\mathrm{R}}$ & Dispersion based on concentration of carrier stream solution, $D^{\mathrm{R}}=C_{\mathrm{m}}^{\mathrm{R}} / C_{\mathfrak{p}}^{\mathrm{R}}$ \\
\hline$L$ & Tube length between injector and nebulizer \\
\hline$R_{\mathrm{i} / \mathrm{a}}$ & $\begin{array}{l}\text { Minimum mass ratio of interferent to analy te species necessary to achieve maximum } \\
\text { interference effect }\end{array}$ \\
\hline$t$ & Time \\
\hline $\boldsymbol{u}$ & Pumping rate, i.e., volume flow rate \\
\hline$V$ & Volume of hypothetical mixing chamber \\
\hline$V_{\mathbf{i}}$ & $\therefore \quad,{ }^{\prime}, \cdots$ \\
\hline
\end{tabular}

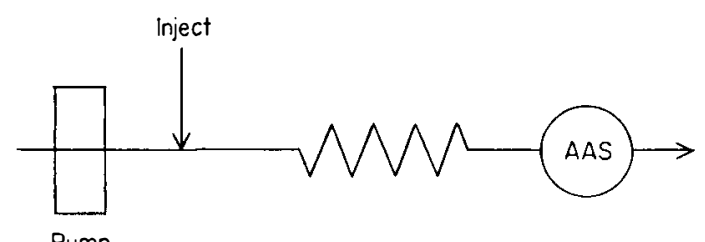

Fig. 1. Flow injection manifold with atomic absorption detector. The pumping rate, $u$, is about $6 \mathrm{ml} \mathrm{min}-1$; connecting tube dimensions are $0.58 \mathrm{~mm}$ i.d. and lengths, $L$, between 3 and $200 \mathrm{~cm}$; the apparent detector volume is $100 \mu \mathrm{l}$.

The use of this model in the design of a flow injection manifold for performing reagent addition and standard addition is described in this paper. Furthermore, it is proposed that the inclusion of a real mixing chamber into the flow system, whose volume is large compared with the hypothetical volume, could provide concentration-time profiles useful for calibration purposes.

\section{EXPERIMENTAL}

\section{Apparatus}

A Gilson Minipuls-2 peristaltic pump, together with an Altex type 201-25 8-port injection valve (having two external sample loops) and $0.58 \mathrm{~mm}$ i.d. tubing formed the basis of the flow injection manifold. A Shandon Southern A3300 atomic absorption spectrometer was used for the studies on reagent 
and standard addition methods and a Perkin-Elmer 290B spectrometer was used for the studies of the concentration profiles for calibration. The spectrometer outputs were monitored either by a chart reconder or a Baird Atomic Datacomp microprocessor data handling accessory.

The flow injection manifold was as shown in Fig. 1. A variety of injection loop volumes ranging from 13 to $500 \mu \mathrm{l}$ and connecting tube lengths from 3 to $200 \mathrm{~cm}$ were used.

\section{Samples, standards and reagents}

The iron samples were prepared by dissolution in hydrochloric acid, as described by Nall et al. [19]. The chromium and magnesium standards were prepared by dilution of stock $1000 \mathrm{mg} \mathrm{l}^{-1}$ solutions (BDH Chemicals). Iron(III) solution (10,000 $\left.\mathrm{mg} \mathrm{l}^{-1}\right)$ was prepared by dissolving the appropriate amount of high-purity iron granules (BCS 149/3) in hydrochloric and nitric acids.

\section{Procedures}

Preliminary experiments. The variation of dispersion as a function of pumping rate, tube length, and volume injected was investigated. The precision of the procedure was evaluated by replicate injections of the standard solution.

Reagent addition method. A carrier stream of iron(III) was used and various volumes of sample and standards were injected. The length of connecting tubing was also varied. For the steel analysis, the tube length was $200 \mathrm{~cm}$ and the volume injected was $50 \mu \mathrm{l}$.

Standard addition method. The samples were used as the carrier stream and various volumes of the standards were injected. Various lengths of connecting tube were investigated. A tube length of $200 \mathrm{~cm}$ and an injection volume of $50 \mu \mathrm{l}$ were used in the analysis of steel samples.

Exponential dilution flask calibration method. A cylindrical glass mixing chamber of volume $8.5 \mathrm{ml}$ was incorporated into the flow line. The inlet was located on a base diameter and the outlet axially at the top [18]. A stream switching method was used to produce a sharp boundary between tri-distilled water and a $2.5 \mathrm{mg} \mathrm{l}^{-1}$. magnesium standard solution. The mixing chamber was stirred by a magnetic follower.

\section{RESULTS AND DISCUSSION}

As the quantitative parameter used in these studies was peak height, the dispersion, $D$, of the system was considered to be the ratio of the steadystate concentration, $C_{\mathrm{m}}$, to the concentration at the peak, $C_{\mathrm{p}}$. This designation of dispersion follows the accepted practice of previous flow injection publications. Thus $D=C_{\mathrm{m}} / C_{\mathrm{p}}$. The value of $V$, the volume of the hypothetical mixing chamber, was calculated for various values of $L$ from the variation of $D$ with $V_{i}$ for each particular value of $L$. On the basis of the 
proposed model, the peak maximum is achieved as the rear boundary of the plug of sample just enters the mixing chamber. This occurs at time $t_{\mathrm{m}}=$ $V_{\mathrm{i}} / V$. Substitution in Eqn. (1) gives

$C_{\mathrm{p}}=C_{\mathrm{m}}\left[1-\exp \left(-V_{\mathrm{i}} / V\right)\right]$

Thus $D=\left[1-\exp \left(-V_{\mathrm{i}} / V\right)\right]^{-1}$

Thus $\ln (1-1 / D)=-V_{\mathrm{i}} / V$ and $\ln [D /(D-1)]=V_{\mathrm{i}} / V$.

Thus a plot of $\ln [D /(D-1)]$, which in practice is calculated as $\ln \left[A_{\mathrm{m}} /\right.$ $\left.\left(A_{\mathrm{m}}-A_{\mathrm{p}}\right)\right]$, vs. $V_{\mathrm{i}}$ has a slope of $1 / V$. For $L$ equal to $200 \mathrm{~cm}$, a least-squares fit of the regression line to the points produced the equation

$\ln \left[A_{\mathrm{m}} /\left(A_{\mathrm{m}}-A_{\mathrm{p}}\right)\right]=0.00488 V_{\mathrm{i}}-0.0143$

The correlation coefficient was 0.999 and the value of $V$ was $205 \mu$ l. Replicate injection of standards had a precision of $0.8 \%$ relative standand deviation based on peak height.

Analysis of the curve shape according to Eqn. (2) produced when a step change in concentration was made close to the nebulizer, gave a virtually constant value of $V$ of $61 \mu \mathrm{l}$ for flow rates between 3.7 and $9.5 \mathrm{ml} \mathrm{min}^{-1}$. This is due to the mode of operation of the nebulizer which will give a steady-state response signal which is a function of flow rate.

\section{Reagent addition method}

If the carrier stream contains a reagent of concentration $C_{\mathrm{m}}^{\mathrm{R}}$ then as the sample plug passes through the "mixing chamber", the reagent concentration varies according to $C^{\mathrm{R}}=C_{\mathrm{m}}^{\mathrm{R}} \exp (-u t / V)$. Thus, at the peak maximum,

$C_{\mathrm{P}}^{\mathrm{R}}=C_{\mathrm{m}}^{\mathrm{R}} \exp \left(-V_{\mathrm{i}} / V\right)$

and, by analogy with the dispersion of the sample (Eqn. 4), the reagent dispersion, $D^{\mathrm{R}}$, is given by

$D^{\mathrm{R}}=C_{\mathrm{m}}^{\mathrm{R}} / C_{\mathrm{P}}^{\mathrm{R}}=\exp \left(-V / V_{\mathrm{i}}\right)$

Thus, combining Eqns. (4) and (6)

$D^{\mathrm{R}}=D /(D-1)$

In the determination of chromium in steel, it is known that the iron exerts a depressive effect on the chromium absorbance. It was found in this study that the depressive effect was constant, provided that the iron-to-chromium mass ratio was greater than $30: 1$. If the most concentrated standard $\left(C_{t}^{\mathrm{s}}\right)$ used in the calibration was $20 \mathrm{mg} \mathrm{l}^{-1} \mathrm{Cr}$, then the relation between dispersion and peak concentration enables the minimum iron concentration at the peak to be calculated. Thus from Eqns. (6) and (7), the minimum concentration of iron in the carrier stream can be calculated as $C_{\mathrm{m}}^{\mathrm{Fe}}=20 \mathrm{X}$ $30 /(D-1)$. This equation can be generalized to give

$C_{\mathrm{m}}^{\mathrm{R}}=C_{\mathrm{t}}^{\mathrm{S}} \times R_{\mathrm{i} / \mathrm{a}} /(D-1)$ 
In this study, a dispersion of 4 was used and thus the concentration of iron added to the carrier was $200 \mathrm{mg} \mathrm{l}^{-1}$ or greater. The results obtained under these conditions for the analysis of some British Chemical Standard steels are given in Table 2 .

\section{Standard addition method}

The sample carrier stream contains the analyte at concentration $C_{\mathrm{x}}$ and thus the concentration at peak maximum when a standard of concentration $C^{S}$ is injected is obtained by combining Eqns. (3) and (5) to give

$C_{\mathrm{p}}=C^{\mathrm{s}}\left[1-\exp \left(-V_{\mathrm{i}} / V\right)\right]+C_{\mathrm{x}} \exp \left(-V_{\mathrm{i}} / V\right)$

The change in concentration, $\Delta C$, which occurs at the peak maximum, $C_{\mathrm{p}}-C_{\mathrm{x}}$, is given by

$\Delta C=C^{\mathrm{s}}\left[1-\exp \left(-V_{\mathrm{i}} / V\right)\right]+C_{\mathrm{x}} \exp \left(-V_{\mathrm{i}} / V\right)-C_{\mathrm{x}}$

Thus $\Delta C=\left(C^{\mathrm{s}}-C_{\mathrm{x}}\right)\left[1-\exp \left(-V_{\mathrm{i}} / V\right)\right]$ and from Eqn. (4), $\Delta C=D^{-1}$ $\left(C^{\mathrm{S}}-C_{\mathrm{x}}\right)$. Thus a plot of $\Delta C$ vs. $C^{\mathrm{s}}$. would intercept the $C^{\mathrm{S}}$ axis at $C_{\mathrm{x}}$. Assuming absorbance to be a linear function of concentration then

$$
\Delta A=(k / D)\left(C^{\mathrm{S}}-C_{\mathrm{x}}\right)
$$

where $\Delta A$ is the observed change in absorbance and $k$ is the proportionality constant relating absorbance and concentration. The method is illustrated in Fig. 2 in which the concentration of the carrier stream was $9.0 \mathrm{mg} \mathrm{l}^{-1}$. The normal use of the standard addition method in a.a.s. is to compensate for interference effects in the samples. For this flow injection method to function likewise, the dispersion must be designed so that interference effects in the sample stream operate to the appropriate extent on the injected standards. This can be done in a manner similar to that outlined in the previous section for the reagent addition method.

From Eqns. (6), (7) and (9),

$C_{\mathrm{p}}=\left(C_{\mathrm{t}}^{\mathrm{s}} / D\right)+C_{\mathrm{x}}(D-1) / D$

Thus the concentration of interferent $C_{\mathrm{p}}^{\mathrm{R}}$ at the peak must be

$C_{\mathrm{p}}^{\mathrm{R}}=R_{\mathrm{i} / \mathrm{a}}\left[\left(C_{\mathrm{t}}^{\mathrm{S}} / D\right)+C_{\mathrm{x}}(D-1) / D\right]$

\section{TABLE 2}

Results of reagent addition and standard addition calibration methods

\begin{tabular}{lccc}
\hline Sample & \multicolumn{2}{c}{ Chromium found (\%) } & $\begin{array}{r}\text { Certified } \\
\text { value (\%) }\end{array}$ \\
\cline { 2 - 3 } & Reagent addition & Standard addition & 5.12 \\
\hline BCS 220/2 & $5.12 \pm 0.06$ & $5.13 \pm 0.02$ & 5.35 \\
BCS 241/2 & $5.34 \pm 0.02$ & $5.34 \pm 0.02$ & 17.4 \\
BCS 261/1 & $17.3 \pm 0.1$ & $17.4 \pm 0.1$ & \\
\hline
\end{tabular}




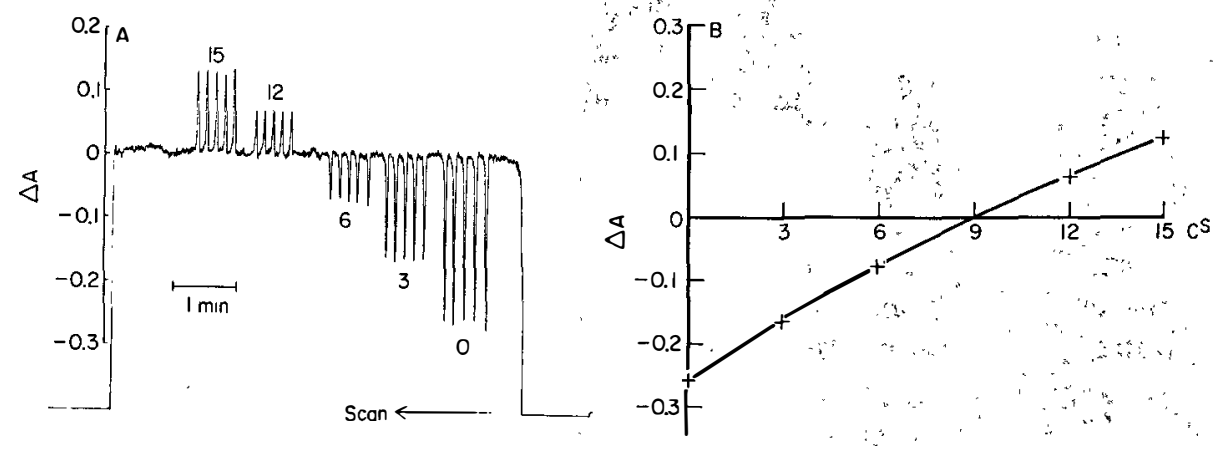

Fig. 2. (A) Chart recording of response for standard addition method. The "'sample" was the carrier stream $\left(9 \mathrm{mg} \mathrm{l}^{-1} \mathrm{Cr}\right.$ ) and the peaks correspond to the injection of solutions containing $0,3,6,12$ and $15 \mathrm{mg} \mathrm{l}^{-1} \mathrm{Cr}$. (B) Plot of results from Fig. 2. A as $\Delta A$ (difference between peak absorbance and carrier stream absorbance) vs. concentration of standard. When $\Delta A=0$, then the concentration corresponds to that in the carrier stream (see Eqn. 10).

and, from Eqns. (6) and (7), the minimum concentration of iron in the carrier stream must be

$C_{\mathrm{m}}^{\mathrm{R}}=R_{\mathrm{i} / \mathrm{a}}\left[C_{\mathrm{t}}^{\mathrm{s}} /(D-1)+C_{\mathrm{x}}\right]$

Equation (11) is thus the general equation for the standard addition method analogous to Eqn. (8) for the reagent addition method."

The conditions used in the experiments described here were $D=4, C_{\mathrm{t}}^{\mathrm{S}}=$ $20 \mathrm{mg} \mathrm{l}^{-1}, R_{\mathrm{t} / \mathrm{a}}(\mathrm{Fe}: \mathrm{Cr})=30$. Thus for a sample containing $10 \mathrm{mg} \mathrm{l}^{-1} \mathrm{Cr}$, the minimum concentration of the interferent, iron, had to be $500 \mathrm{mg} \mathrm{l}^{-1}$. As the samples did not contain the appropriate iron to chromium concentration ratio, sufficient iron was added so that the sample carrier streams contained at least $500 \mathrm{mg} \mathrm{l}^{-1}$ iron. The results obtained by this method for the BCS steels are shown in Table 2.

\section{Exponential dilution flask calibration method}

The concentration-time profile generated by a real mixing chamber of volume $V_{\mathrm{m}}$ is given from Eqn. (1): $C=C_{\mathrm{m}}\left[1-\exp \left(-u t / V_{\mathrm{m}}\right)\right]$. An analysis of the curve shape, produced at a flow rate, $u$, of $5.1 \mathrm{ml} \mathrm{min}^{-1}$, showed that the mixing chamber behaved as though $V_{\mathrm{m}}$ were $7.2 \mathrm{ml}$. This value was used in subsequent calculations. The curve produced by the instrument when the concentration was stepped from zero to $2.5 \mathrm{mg} \mathrm{l}^{-1}$ is shown in Fig. 3; the steady-state signal for this concentration corresponded to $16.6 \mathrm{~cm}$ on the chart paper. The curve obtained from

$A=16.6[1-\exp (-5.1 t / 60 \times 7.2)]$

is also shown in the Fig. 3. The good agreement which is obtained shows that the dispersion of the real mixing chamber completely dominates all other sources of dispersion in the system. Thus, if a sample solution is introduced 


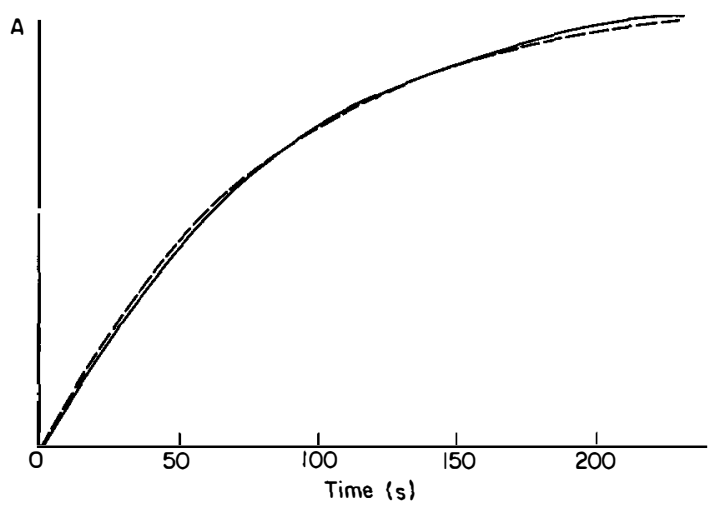

Fig. 3. Absorbance-time relationships: (-) produced when a step change in concentration occurred just prior to the mixing chamber; (-.) calculated according to Eqn. (12).

into the instrument at the same flow rate as the exponential concentration profile was produced, a characteristic time value may be obtained from the $A-t$ plot which can then be converted to a concentration by substitution into Eqn. (1). The results of some preliminary experiments with magnesium as the test element are given in Table 3.

\section{CONCLUSIONS}

In addition to the advantages of using flow injection sample introduction methods for atomic absorption spectrometry, which have already been described in the literature, the results of the present study indicate that the precise dispersion characteristics of flow injection manifolds can be used as a substitute for volumetric manipulation and that flow injection analogues of "reagent" addition (matching standards to samples) and standard addition methods of calibration may be devised. This standard addition method, in particular, has two advantages: (a) the same standard solutions are used for a range of samples, thus considerably reducing the amount of volumetric manipulation necessary; and (b) an interpolative procedure is provided for obtaining unknown concentrations, which is more accurate than the normal extrapolative method.

Although it has been shown that the simplified model for the dispersion effects observed proposed here (the single hypothetical mixing chamber) is lyte. In the g.s.a.m. calculations, these factors may be considered as a pseudoapplicable to flow injection manifolds coupled to atomic absorption spec-

\section{TABLE 3}

Results for exponential dilution flask calibration method

\begin{tabular}{lllllll}
\hline Real conc. (mg l & \\
Conc. found $\left(\mathrm{mg} \mathrm{l}^{-1}\right)$ & 0.125 & 0.25 & 0.5 & 1.0 & 1.5 & 2.0 \\
Error $(\%)$ & 0.128 & 0.255 & 0.52 & 1.04 & 1.55 & 2.07 \\
\hline
\end{tabular}


trometers, it may be that this is due to the flow rates and detector behaviour peculiar to a.a.s., and that the model may not have general applicability. It is difficult to examine previously published data to see if this model fits other flow injection systems, as very few publications give sufficient information concerning peak shape, dispersion, etc. However, a preliminary examination of some of Rưžčka's and Hansen's results [18] (particularly when Figs. 10, 11 and 12 of ref. 18 are examined) for a solution spectrophotometric system with detector volume $18 \mu \mathrm{l}$, pumping rates 0.5 and $1.5 \mathrm{ml} \mathrm{min} \mathrm{m}^{-1}$, and tube lengths $(0.5 \mathrm{~mm}$ i.d. $)$ up to $200 \mathrm{~cm}$, is very encouraging in this respect.

It is hoped to provide further details of the new model, including consideration of some of the relevant time parameters of peaks, and of the exponential dilution flask calibration method as well as examination of other flow injection-based methods of generating concentration gradients that could be utilized for calibration purposes, in future publications. It has recently been reported [20] that the tail section of a single injection can be used empirically in this way to provide a method of "electronically" reconstructing a calibration curve. Thus, "it is hoped that the present outline will inspire further thoughts about the undiscovered possibilities which f.i.a. gradient techniques have to offer" [20].

A. B. Idris and J. M. H. Appleton gratefully acknowledge financial support from the National University of Malaysia and the Zimbabwe Government Department of Manpower Training and Social Services, respectively.

\section{REFERENCES}

1 J. Růžička and E. H. Hansen, Anal. Chim. Acta, 78 (1975) 145.

2 T. Uchida, I. Kojima and C. Iida, Anal. Chim. Acta, 116 (1980) 205.

3 R. C. Fry, S. J. Northway and M. B. Denton, Anal. Chem., 50 (1978) 1719.

4 W. R. Wolf and K. K. Stewart, Anal. Chem., 51 (1979) 1201.

5 N. Yoza, Y. Aoyagi, S. Ohashi and A. Tateda, Anal. Chim. Acta, 111 (1979) 163.

$6 \mathrm{~K}$. Fukamachi and N. Ishibashi, Anal. Chim. Acta, 119 (1980) 383.

7 E. A. G. Zagatto, F. J. Krug, H. Bergamin F⿳丷, S. S. Jфrgensen and B. F. Reis, Anal. Chim. Acta, 104 (1979) 279.

8 B. F. Reis, A. O. Jacintho, J. Mortatti, F. J. Krug, E. A. G. Zagatto, H. Bergamin Fo and L. C. R. Pessenda, Anal. Chim. Acta, 123 (1981) 221.

9 E. A. G. Zagatto, A. O. Jacintho, L. C. R. Pessenda, F. J. Krug, B. F. Reis and H. Bergamin $F^{\mathbf{Q}}$, Anal. Chim. Acta, 125 (1981) 37.

10 W. D. Basson and J. F. van Staden, Fresenius Z. Anal. Chem., 302 (1980) 370.

11 B. D. Mindel and B. Karlberg, Lab. Pract., July (1981) 719.

12 J. F. Tyson, Anal. Proc., 18 (1981) 542.

13 J. F. Tyson and A. B. Idris, Analyst, 106 (1981) 1125.

14 S. Greenfield, Ind. Res. Dev., August (1981) 140.

15 A. O. Jacintho, E. A. G. Zagatto, H. Bergamin F $^{\circ}$, F. J. Krug, B. F. Reis, R. E. Bruns and B. R. Kowalski, Anal. Chim. Acta, 130 (1981) 243.

16 O. Åström, Anal. Chem., 54 (1982) 190.

17 J. T. Vanderslice, K. K. Stewart, A. G. Rosenfeld and D. J. Higgs, Talanta, 28 (1981) 11. 18 J. Rưžčcka and E. H. Hansen, Anal. Chim. Acta, 99 (1978) 37.

19 W. R. Nall, D. Brumhead and R. Whitham, Analyst, 100 (1975) 555.

20 S. Olsen, J. Rư̌̌ička and E. H. Hansen, Anal. Chim. Acta, 136 (1982) 101. 